\title{
Conductas de ingestión en bovinos de la raza Nelore recibiendo altas concentraciones de glicerina cruda
}

\section{Feeding behavior of Nellore cattle fed high concentrations of crude glycerin}

\author{
Eric Haydt Castello Branco van Cleef $^{*}$; Jane Maria Bertocco Ezequiel²; \\ Rene Mauricio Patiño Pardo ${ }^{3}$; Andre Pastori D`Aurea ${ }^{4}$; David Attuy Vey da Silva ${ }^{5}$; \\ Flavia de Oliveira Scarpino van $\mathrm{Cleef}^{6}$
}

\section{Resumen}

El objetivo de este estudio fue evaluar la inclusión de hasta $30 \%$ de glicerina cruda en dietas para bovinos de la raza Nelore y su efecto sobre parámetros del comportamiento ingestivo. Se utilizaron 30 animales con $277.7 \mathrm{~kg}$ de PV y 18 meses de edad, los cuales fueron mantenidos en confinamiento en corrales individuales durante 103 días (21 de adaptación y 82 de colecta de datos). Los animales fueron divididos en bloques (peso inicial) y sometidos a los siguientes tratamientos: G0; G7.5; G15; G22.5 y G30, correspondientes al grupo control, $7.5,15,22.5$ y $30 \%$ de glicerina cruda en la materia seca de la dieta, respectivamente. Las conductas del comportamiento (alimentación, ocio, rumia, número de masticaciones merícicas, eficiencias de alimentación y rumia) se evaluaron durante tres días. Los datos fueron analizados según un diseño en bloques al azar, realizando análisis de contrastes y observando la significancia de los efectos lineal y cuadrático y del tratamiento control $\times$ tratamientos con glicerina. La inclusión de hasta $30 \%$ de glicerina cruda en la dieta de los bovinos de la raza Nelore alteró la eficiencia de alimentación, expresada en $\mathrm{g} \mathrm{FDN} \mathrm{h}^{-1}$, la eficiencia de rumia en relación al FDN, el tiempo y número de masticaciones merícicas por bolo ruminal, facilitando la ingestión de los alimentos, e influenciando directamente el tiempo de alimentación.

Palabras-clave: Biodiesel, coproducto, masticaciones merícicas, ocio, rumia

\begin{abstract}
The objective of this study was to evaluate the inclusion of up to $30 \%$ crude glycerin in Nellore cattle diets and its effects on feeding behavior parameters. It were used 30 animals with $277.7 \mathrm{~kg} \mathrm{BW}$ and 18 months old, which were kept in feedlot in individual pens during 103 days (21 adaptation and 82 data collection). The animals were assigned (initial weight) in blocks and submitted to the following treatments: G0; G7.5; G15; G22.5; and G30, corresponding to control group, 7.5, 15, 22.5, and 30\% crude glycerin in the diet dry matter, respectively. The feeding behavior (feeding, idle, ruminating, number of chews, feeding efficiency and ruminating efficiency) were evaluated for three days. Data were analyzed
\end{abstract}

\footnotetext{
${ }^{1}$ Discente de Pós-doutorado, Dept ${ }^{\circ}$ de Zootecnia, Faculdade de Ciências Agrárias e Veterinárias, Universidade Estadual Paulista "Júlio de Mesquita Filho", UNESP, Jaboticabal, SP. E-mail: ericvancleef@gmail.com

2 Prof $^{a}$ Dra $^{\mathrm{a}}$, Dept $^{\mathrm{o}}$ de Zootecnia, UNESP, Jaboticabal, SP. E-mail: janembe_fcav@yahoo.com.br

${ }^{3}$ Prof. Dr., Faculdad de Ciencias Agropecuarias, Universidad de Sucre, Sincelejo, Sucre, Colômbia. E-mail: rene.patino@unisucre. edu.co

${ }^{4}$ Pesquisador, Premix Ltda, Patrocínio Paulista, SP, Brasil. E-mail: andre.daurea@premix.com.br

5 Discente de Mestrado em Medicina Veterinária, UNESP, Jaboticabal, SP. E-mail: davidattuy@hotmail.com

${ }^{6}$ Discente de Mestrado em Zootecnia, UNESP, Jaboticabal, SP. E-mail: flascarpino@hotmail.com

* Autor para correspondência
} 
as a completely randomized block design, analyzing contrasts and observing the significance of linear, quadratic and control treatment $\times$ glycerin treatments effects. The inclusion up to $30 \%$ crude glycerin in diets of Nellore cattle altered the feeding efficiency, expressed in $\mathrm{g} \mathrm{NDF} \mathrm{h}^{-1}$, the ruminating efficiency relative to NDF, the time and number of chews per ruminal bolus, facilitating the feed ingestion and directly influencing the time spent on feeding.

Key words: Biodiesel, co-product, idle, rumination chews, rumination

\section{Introducción}

En el actual proceso de intensificación de la ganadería de carne brasileña, se verifica el incremento en la práctica del confinamiento como estrategia alimenticia o alternativa de terminación de animales.

En todo sistema de producción en confinamiento, los costos de adquisición de ingredientes para la fabricación de raciones son muy elevados, principalmente debido a la alta proporción de concentrados (LOPES; MAGALHÃES, 2005). Una de las soluciones para minimizar ese problema sería la introducción de subproductos de la agroindustria en las dietas de los animales (DILORENZO; GALYEAN, 2010; TREJO et al., 2010).

Con la situación actual del biodiesel y la actual legislación del gobierno brasileño, que obliga a la adición de $5 \%$ de ese combustible al diésel común, hay un excedente de producción de glicerina que debe ser absorbido, de manera ambientalmente viable e económicamente correcta. Los subproductos de la agroindustria del biodiesel (tortas, harinas y glicerina) surgen como alternativas viables para la alimentación de rumiantes (ABDALLA et al., 2008).

La introducción de la glicerina en la dieta de vacunos de carne y lecheros viene siendo investigada en los últimos años y parece ser económicamente viable cuando el producto es incluido hasta $15 \%$ de la dieta (PARSONS; SHELOR; DROUILLARD, 2009; MACH; BACH; DEVANT, 2009; DONKIN et al., 2009; CHUNG et al., 2007). Los resultados también indica que se puede utilizar ese coproducto como sustituto parcial al maíz en dietas de animales de elevada producción (DONKIN, 2008).

Las actividades diarias son caracterizadas por tres comportamientos básicos: alimentación, rumia y ocio (PAZDIORA et al., 2011), siendo los rumiantes capaces de adaptarse a diversas condiciones de alimentación, manejo y ambiente, y modifican los parámetros del comportamiento ingestivo para alcanzar y mantener determinado nivel de consumo, compatible con las exigencias nutricionales (CARDOSO et al., 2006).

Cuando se introduce un nuevo ingrediente en dietas para animales es importante estudiar los efectos de esa acción sobre las conductas de ingestión, ya que de esto depende la respuesta de los animales en términos productivos (SILVA et al., 2005; MENDONÇA et al., 2004).

En ese sentido, el objetivo de este ensayo fue evaluar la inclusión de hasta $30 \%$ de glicerina cruda en la ración para bovinos en terminación y su efecto sobre el comportamiento ingestivo.

\section{Materiales y Métodos}

Elexperimento fue conducido en el confinamiento experimental perteneciente al Departamento de Zootecnia de la Facultad de Ciencias Agrarias y Veterinarias/Unesp, Campus de Jaboticabal, el cual posee 30 corrales individuales semicubiertos, con piso en concreto, y comederos y bebederos individuales.

Fueron utilizados 30 animales de la raza Nelore, no castrados, con peso corporal de $277.7 \mathrm{~kg}$ y aproximadamente 18 meses de edad, los cuales fueron distribuidos a los tratamientos según un diseño en bloques al azar, con cinco tratamientos y seis repeticiones.

Las cinco raciones experimentales isoproteicas (12.2\% PB) e isoenergéticas (2.5 Mcal EM kg-1 MS) 
fueron formuladas de acuerdo al NRC (1996) y ofrecidas guardando una relación forraje:concentrado de 30:70. Las dietas o tratamientos fueron denominadas como: G0; G7.5; G15; G22.5 y G30, correspondientes al grupo control, $7.5,15,22.5$ y $30 \%$ de glicerina cruda en la materia seca de la dieta, respectivamente. La composición de los ingredientes y de las dietas se presenta en las Tablas 1 y 2 , respectivamente.

Tabla 1. Matéria seca (MS), proteína cruda (PC), extrato etéreo (EE) e energia metabolizável (EM) dos alimentos utilizados na formulação das dietas experimentais.

\begin{tabular}{|c|c|c|c|c|c|c|}
\hline \multirow{2}{*}{ Ingredientes } & MS & $\mathrm{PC}$ & $\mathrm{EE}$ & FDN & FDA & \multirow{2}{*}{$\begin{array}{c}\text { EM } \\
\text { Mcal kg-1 MS }\end{array}$} \\
\hline & $(\%)$ & \multicolumn{4}{|c|}{$\% \mathrm{MS}$} & \\
\hline Ensilaje de maíz & 30,92 & 7,26 & 3,16 & 55,41 & 30,63 & 2,21 \\
\hline Maíz & 87,94 & 9,11 & 4,07 & 13,98 & 4,08 & 3,10 \\
\hline Harina de girassol & 91,10 & 31,50 & 1,50 & 42,36 & 34,90 & 2,17 \\
\hline Glicerina cruda $^{1}$ & 90,00 & 0,00 & 0,50 & 0,00 & 0,00 & 3,00 \\
\hline Cascarilla de soya & 89,80 & 11,65 & 1,60 & 68,40 & 50,52 & 2,42 \\
\hline Sal común & 99,00 & 0,00 & 0,00 & 0,00 & 0,00 & 0,00 \\
\hline Carbonato de cálcio & 99,00 & 0,00 & 0,00 & 0,00 & 0,00 & 0,00 \\
\hline Fosfato bicálcico & 99,00 & 0,00 & 0,00 & 0,00 & 0,00 & 0,00 \\
\hline
\end{tabular}

Análises realizadas no Laboratório de Ingredientes e Gases Poluentes da Unidade Animal de Estudos Digestivos e Metabólicos da FCAV/Unesp. ${ }^{1}$ Caramuru Alimentos S. A.

Fuente: Elaboración de los autores.

Tabla 2. Porcentaje de los ingredientes y estimativa de la composición bromatológica de los tratamientos experimentales (\% MS).

\begin{tabular}{lccccc}
\hline \multirow{2}{*}{ Ingredientes } & \multicolumn{5}{c}{ Tratamientos } \\
\cline { 2 - 5 } & $\mathrm{G} 0^{1}$ & $\mathrm{G} 7,5^{2}$ & $\mathrm{G} 15^{3}$ & $\mathrm{G} 22,5^{4}$ & $\mathrm{G} 30^{5}$ \\
\hline Ensilaje de maíz & 30,00 & 30,00 & 30,00 & 30,00 & 30,00 \\
Maíz & 35,00 & 25,50 & 18,00 & 12,50 & 5,00 \\
Harina de girasol & 14,60 & 17,8 & 21,30 & 24,90 & 28,4 \\
Cascarilla de soya & 19,20 & 18,05 & 14,55 & 8,90 & 5,45 \\
Glicerina cruda & 0,00 & 7,50 & 15,00 & 22,50 & 30,00 \\
Sal común & 0,50 & 0,50 & 0,50 & 0,50 & 0,50 \\
Carbonato de calcio & 0,70 & 0,65 & 0,55 & 0,70 & 0,65 \\
Fosfato bicálcico & 0,00 & 0,10 & 0,10 & 0,00 & 0,00 \\
\hline & & & Composición & & \\
\hline PC (\% MS) & 12,20 & 12,21 & 12,22 & 12,20 & 12,21 \\
EM (Mcal kg-1 MS) & 2,53 & 2,52 & 2,52 & 2,53 & 2,52 \\
EE (\%) & 2,90 & 2,58 & 2,31 & 2,09 & 1,81 \\
FDN (\%) & 40,83 & 40,07 & 38,11 & 35,01 & 33,08 \\
FDA (\%) & 25,41 & 25,56 & 24,71 & 22,89 & 22,06 \\
HEM (\%) & 15,42 & 14,51 & 13,41 & 12,12 & 11,02 \\
Ca (\%) & 0,56 & 0,56 & 0,55 & 0,57 & 0,55 \\
P (\%) & 0,32 & 0,32 & 0,35 & 0,34 & 0,34 \\
\hline
\end{tabular}

${ }^{1}$ Tratamiento control sin adición de glicerina; ${ }^{2}$ Adición de 7,5\% de glicerina en la MS de la dieta; ${ }^{3}$ Adición de $15 \%$ de glicerina en la MS de la dieta; ${ }^{4}$ Adición de 22,5\% de glicerina en la MS de la dieta; ${ }^{5}$ Adiçión de $30,0 \%$ de glicerina en la MS de la dieta.

Fuente: Elaboración de los autores. 
La glicerina cruda utilizada presentó una concentración de glicerol de $86 \%, 90 \%$ de materia seca, $6 \%$ de sales y menos de $0.01 \%$ de metanol. El forraje utilizado fue ensilaje de maíz y el concentrado compuesto por maíz, cascarilla de soya $\mathrm{y}$ harina de girasol.

Los animales fueron confinados por 103 días, siendo los primeros 21 días destinados a la adaptación de los animales, y los restantes 82 días para las evaluaciones y colecta de datos. Los animales fueron pesados al inicio del periodo de adaptación, para la división de los mismos dentro de los bloques.

El alimento fue ofrecido dos veces al día, a las $08 \mathrm{~h} 00$ y $18 \mathrm{~h} 00$. El concentrado y el ensilaje fueron pesados separadamente y mezclados a la glicerina al momento de la oferta, en cada una de las raciones diarias. Antes de la primera oferta diaria de alimento, las sobras del día anterior eran pesadas, y una muestra correspondiente al 10\%, con la finalidad de ajustar los consumos diarios de materia seca. Las muestras de las sobras, tomadas diariamente, eran combinadas, dando origen a las muestras compuestas, por semana y por animal, en las cuales fueron determinadas las proporciones de materia seca, según metodología de Silva e Queiroz (2002), y fibra en detergente neutro, de acuerdo con Goering e Van Soest (1970), para calcular el consumo de nutrientes.

La colecta de datos para la evaluación del comportamiento ingestivo ocurrió durante tres días de observaciones, en intervalos de 27 días. En cada periodo, la observación de las conductas fue realizada de modo focal directo, con intervalos de 10 minutos (SILVA; SILVA; PRADO, 2006). Fueron utilizados tres observadores entrenados (una para cada 10 animales), cada seis horas, totalizando, 24 horas. Fueron registrados los tiempos diarios invertidos en el consumo de alimento, rumiando echado o en pie y ocio o descanso, echado o de pie.

La media del número de masticaciones merícicas por bolo ruminal (NMB) y el tiempo invertido en la masticación merícica por bolo ruminal (TMB) fueron obtenidos a través de observaciones por animal rumiando en cada periodo de evaluación, totalizando al menos 12 observaciones por animal, por periodo. Para el registro del TMB se utilizó cronómetro digital.

Utilizando la metodología propuesta por Bürger et al. (2000), fueron calculados: TAL (h día ${ }^{-1}$ ), tiempo de alimentación; TRU (h día $\left.\mathrm{a}^{-1}\right)$, tiempo de rumia; MMtb (s bolo-1), tiempo de masticaciones merícicas por bolo ruminal; $\mathrm{MMnb}\left(\mathrm{n}^{\circ}\right.$ bolo-1 $)$, número de masticaciones merícicas por bolo; TOE ( $\mathrm{h}$ día $^{-1}$ ), el tiempo de ocio o descanso en pie; el tiempo de ocio o descanso echado y las siguientes relaciones: EAL (eficiencia de alimentación) = CMS/TAL $\left(\mathrm{g} \mathrm{MS} \mathrm{h}^{-1}\right)$; ERU (eficiencia de rumia) $=$ $\mathrm{CMS} / \mathrm{TRU}\left(\mathrm{g} \mathrm{MS} \mathrm{h}^{-1}\right)$; $\mathrm{ERU}_{\mathrm{FDN}}$ (eficiencia de rumia del FDN $)=$ CFDN/TRU $\left(\right.$ g FDN h$\left.^{-1}\right)$; TMT (tiempo total de masticación $)=$ TAL + TRU $\left(\right.$ h día $\left.^{-1}\right) ; \mathrm{BOL}$ (número de bolos ruminales) $=$ TRU/MMtb $\left(n^{\circ}\right.$ día ${ }^{1}$ ); MMnd (masticaciones merícicas) = BOLMMnb $\left(\mathrm{n}^{\mathrm{o}}\right.$ día $\left.^{-1}\right)$; y TOT (tiempo de ocio total $)=$ TOE + TOD (h día $\left.{ }^{-1}\right)$.

Los datos fueron analizados según un diseño en bloques al azar, con cinco tratamientos y seis repeticiones utilizando el procedimiento MIXED do programa estadístico SAS, versión 9.1. En el modelo se incluyeron los efectos de los factores tratamiento, bloque y periodo de observación. Fue realizado análisis de contrastes observando la significancia de los modelos lineal y cuadrático y del tratamiento control $\times$ tratamientos con glicerina.

\section{Resultados y Discusión}

Se observó que el tiempo dedicado al consumo de alimento fue influenciado $(\mathrm{P}<0.05)$ por la inclusión de glicerina en la dieta, siendo mayor el tiempo invertido cuando se incluyó glicerina en la dieta (Tabla 3). Este comportamiento pudo deberse, posiblemente, a la modificación física de la dieta promovida por la glicerina, por la agregación de las partículas de la ración. 
Tabla 3. Tiempos invertidos en consumo de alimento (TAL), rumia de pie (TRE), rumia echado (TRD), rumia total (TRU), ocio en pie (TOE), ocio echado (TOD) e ocio total (TOT), por bovinos alimentados con dietas con inclusión de hasta $30 \%$ de glicerina cruda.

\begin{tabular}{|c|c|c|c|c|c|c|c|c|c|}
\hline \multirow{2}{*}{$\begin{array}{c}\text { Actividad } \\
\left(\mathrm{h} \mathrm{día}^{-1}\right)\end{array}$} & \multicolumn{5}{|c|}{ Glicerina cruda (\%) } & \multicolumn{3}{|c|}{ Contrastes } & \multirow{2}{*}{$\mathrm{RMSE}^{4}$} \\
\hline & G0 & $\mathrm{G} 7,5$ & G15 & G22,5 & G30 & $\mathrm{L}^{1}$ & $\mathrm{C}^{2}$ & $0 \times \mathrm{GLI}^{3}$ & \\
\hline TAL & 2,20 & 2,70 & 2,60 & 2,80 & 2,60 & NS & NS & $*$ & 0,80 \\
\hline TER & 1,20 & 0,80 & 0,90 & 1,00 & 0,70 & NS & NS & NS & 0,80 \\
\hline TRD & 3,30 & 4,50 & 4,00 & 3,60 & 3,60 & NS & NS & NS & 1,30 \\
\hline TRU & 4,50 & 5,30 & 4,90 & 4,60 & 4,30 & NS & NS & NS & 1,30 \\
\hline TOE & 7,30 & 5,80 & 6,70 & 7,50 & 7,30 & NS & NS & NS & 2,50 \\
\hline TOD & 10,00 & 10,30 & 9,90 & 9,20 & 9,80 & NS & NS & NS & 2,50 \\
\hline TOT & 17,30 & 16,10 & 16,60 & 16,70 & 17,10 & NS & NS & NS & 1,80 \\
\hline
\end{tabular}

${ }^{1}$ Lineal. ${ }^{2}$ Cuadrático. ${ }^{3}$ Tratamiento control $\times$ tratamientos con glicerina. ${ }^{4}$ Raiz cuadrada del error medio. $*<0,05 ; * *<0,01, \mathrm{NS}=\mathrm{No}$ significativo.

Fuente: Elaboración de los autores.

Elam et al. (2008) trabajaron en fase de acabado en confinamiento, testando la sustitución parcial de maíz por $10 \%$ de glicerina en la materia seca de la ración, y observaron mayor tiempo invertido en el consumo de alimento. De otra parte, Barroso et al. (2010), al evaluar las conductas de ingestión de novillas suplementadas a pasto con niveles crecientes de glicerina cruda en la ración $(0,3.33,6.66$, y $9.99 \%$ en la materia seca de la ración), verificaron que el tiempo de permanencia en los comederos presentó efecto $(\mathrm{P}<0.01)$ lineal decreciente con el aumento en la inclusión de glicerina. Los autores explicaron que posiblemente ese hecho se debió a que la glicerina cruda es viscosa y que cuando se mezcla a la ración facilita la aglomeración de las partículas y la deglución del suplemento, cuando se adicionan mayores concentraciones de glicerina.

En relación al tiempo de ocio o descanso, tanto total (TOT), echado (TOD) o en pie (TOE), la inclusión de glicerina cruda en la dieta no promovió alteración alguna $(\mathrm{P}>0.05)$, así como el tiempo total de rumia (TRU) y el tiempo de rumia en pie (TRE) y el tiempo de rumia echado (TRD). La última variable fue asociada al consumo de fibra en detergente neutro (CFDN) por Missio et al. (2010), al evaluar diferentes niveles de concentrado $(22,40,59$ y $79 \%)$ en la dieta de bovinos terminados en confinamiento.
Los autores verificaron que el tiempo de rumia echado y de rumia total disminuyeron linealmente $(\mathrm{P}<0.01)$ con el incremento de concentrado en la dieta, principalmente debido a la reducción lineal $(\mathrm{P}<0.01)$ del consumo de FDN. En este trabajo, la media del tiempo de rumia echado $\mathrm{TRD}^{-1}$ fue de $4.25 \mathrm{~h} \mathrm{día}^{-1}$ para los tratamientos que tenían $7.5 \mathrm{y}$ $15 \%$ de glicerina cruda en la ración y de $3.5 \mathrm{~h} \mathrm{día}^{-1}$ en promedio para las demás variables.

Barroso et al. (2010) observaron efecto cuadrático $(\mathrm{P}<0.01)$ para el tiempo de rumia, con un valor máximo para el nivel de $0.99 \%$ de inclusión de glicerina, trabajando con bovinos en pastoreo. Los autores sugirieron que la glicerina, en niveles más bajos de oferta, provoca aumento en el consumo de MS, aumentando de esa manera el tiempo de pastoreo y consecuentemente el tiempo de rumia. Sin embargo, Santana Junior et al. (2013) suplementaron vacas lactantes en pastoreo con concentrado incluyendo hasta $18 \%$ de glicerina, y no observaron ningún efecto sobre las variables del comportamiento de los animales.

La eficiencia de alimentación, expresada en $g$ $\mathrm{MS} \mathrm{h}^{-1}$ fue mayor $(\mathrm{P}<0.01)$ para las raciones con glicerina cruda en relación a la ración control (Tabla 4). Probablemente, la reducción lineal $(\mathrm{P}<0.01)$ de la ingestión de $\mathrm{FDN} \mathrm{h}^{-1}$ haya contribuido bastante, 
ya que hubo aumento de $49 \%$ en la $\mathrm{EAL}_{\mathrm{FDN}}$ entre la eficiencia alimenticia con que el animal capta el los tratamientos G0 y G30. Otro hecho está alimento está directamente relacionada al tiempo relacionado al tiempo destinado a la alimentación, de consumo y al peso específico del alimento que fue mayor para las dietas con glicerina cruda y consumido.

Tabla 4. Consumos de MS (CMS) y de FDN (CFDN), eficiencia de alimentación de MS (EAL) e de FDN (EAL $\left.{ }_{\mathrm{FDN}}\right)$, eficiencia de rumia de la dieta total (ERU) e eficiencia de rumia da FDN (ERU ${ }_{\text {FDN }}$ ) de bovinos alimentados con dietas con inclusión de hasta $30 \%$ de glicerina cruda.

\begin{tabular}{|c|c|c|c|c|c|c|c|c|c|}
\hline \multirow{2}{*}{ Ítem } & \multicolumn{5}{|c|}{ Glicerina cruda (\%) } & \multicolumn{3}{|c|}{ Contrastes } & \multirow{2}{*}{$\mathrm{RMSE}^{4}$} \\
\hline & G0 & G7,5 & G15 & $\mathrm{G} 22,5$ & G30 & $\mathrm{L}^{1}$ & $\mathrm{C}^{2}$ & $0 \times \mathrm{GLI}^{3}$ & \\
\hline CMS $\left(\mathrm{kg} \mathrm{d}^{-1}\right)$ & 8,96 & 7,81 & 8,49 & 8,75 & 7,79 & NS & NS & NS & 0,38 \\
\hline CFDN $\left(\mathrm{kg} \mathrm{d}^{-1}\right)$ & 4,51 & 4,58 & 3,39 & 3,40 & 2,71 & $* *$ & NS & $* *$ & 0,22 \\
\hline $\operatorname{EAL}\left(\mathrm{g} \mathrm{MS} \mathrm{h}^{-1}\right)$ & 5110 & 3070 & 3440 & 3700 & 3540 & NS & $*$ & $* *$ & 1,97 \\
\hline $\mathrm{EAL}_{\mathrm{FDN}}\left(\mathrm{g} \mathrm{FDN} \mathrm{h}^{-1}\right)$ & 2430 & 1780 & 1370 & 1400 & 1230 & $* *$ & $*$ & $* *$ & 0,68 \\
\hline ERU $\left(\mathrm{g} \mathrm{MS} \mathrm{h}^{-1}\right)$ & 2400 & 1550 & 1930 & 2010 & 2020 & NS & NS & $*$ & 0,80 \\
\hline $\mathrm{ERU}_{\mathrm{FDN}}\left(\mathrm{g} \mathrm{FDN} \mathrm{h}^{-1}\right)$ & 1170 & 920 & 760 & 770 & 700 & $* *$ & NS & $* *$ & 0,32 \\
\hline
\end{tabular}

${ }^{1}$ Lineal. ${ }^{2}$ Cuadrático. ${ }^{3}$ Tratamiento control $\times$ tratamientos con glicerina. ${ }^{4}$ Raiz cuadrada del error medio. $*<0,05 ; * *<0,01, \mathrm{NS}=\mathrm{No}$ significativo.

Fuente: Elaboración de los autores.

La inclusión de glicerina cruda presentó efecto $(\mathrm{P}<0.05)$ sobre la eficiencia alimenticia de rumia, expresada en $\mathrm{g} \mathrm{MS} \mathrm{h}{ }^{-1}$, independiente de la dosis de glicerina mínima ofrecida, sin embargo cuando se expresó en g FDN h-1 aumentó linealmente $(\mathrm{P}<0.01)$. El aumento de la ERU $\mathrm{FDN}_{\mathrm{F}}$ está asociado al menor consumo de FDN observado en el presente estudio.

El tiempo de masticación total (TMT), el número de bolos ruminales (BOL) y las masticaciones merícicas (MMnd) no presentaron diferencias con la inclusión creciente de glicerina cruda en la dieta (Tabla 5), sin embargo el número (MMnb) de masticaciones merícicas por bolo ruminal disminuyeron linealmente $(\mathrm{P}<0.01)$.

Las alteraciones observadas en el tiempo y número de masticaciones se deben a que la glicerina es líquida a temperatura ambiente y por ser rápidamente fermentada en el rumen, reduce en 50 a $70 \%$ su volumen en 4 horas (DONKIN, 2008), y también porque sustituyó al maíz, el cual posee elevada densidad. Otro factor que posiblemente contribuyó para ese resultado fue la disminución en la cantidad de cascarilla de soya y el aumento de la harina de girasol en las dietas con inclusión creciente de glicerina cruda, lo que también contribuyó para la forma física de las raciones.

Missio et al. (2010) atribuyeron la reducción del número de masticaciones por bolo ruminal a las características químicas de las dietas que contenían cantidades crecientes de concentrado, y a pesar de haber correlacionado el número de masticaciones por bolo ruminal con el tiempo de masticaciones por bolo ruminal, este parámetro no fue influenciado $(\mathrm{P}>0.05)$ por la concentración de concentrado en la dieta.

A pesar de los efectos observados sobre las variables del comportamiento ingestivo, el peso final, la ganancia de peso y la eficiencia alimenticia no fueron influenciadas $(\mathrm{P}>0.05)$ por los tratamientos (Tabla 6). 
Tabla 5. Tiempo de masticación total (TMT), número de bolos ruminales (BOL), del número de masticaciones merícicas por día (MMnd), número de masticaciones merícicas por bolo ruminal (MMnb) y del tiempo invertido por bolo ruminal (TM) de bovinos alimentados con dietas con inclusión de hasta 30\% de glicerina cruda.

\begin{tabular}{|c|c|c|c|c|c|c|c|c|c|}
\hline \multirow{2}{*}{ Ítem } & \multicolumn{5}{|c|}{ Glicerina cruda $(\%)$} & \multicolumn{3}{|c|}{ Contrastes } & \multirow{2}{*}{$\mathrm{RMSE}^{4}$} \\
\hline & G0 & G7,5 & G15 & G22,5 & G30 & $\mathrm{L}^{1}$ & $\mathrm{C}^{2}$ & $0 \times \mathrm{GLI}^{3}$ & \\
\hline $\begin{array}{l}\text { TMT }^{6} \\
\left(\text { h día }^{-1}\right)\end{array}$ & 6,70 & 8,00 & 7,40 & 7,40 & 6,90 & NS & NS & NS & 1,80 \\
\hline $\begin{array}{l}\text { BOL } \\
\left(n^{\circ} \text { día }^{-1}\right)\end{array}$ & 258,40 & 313,40 & 309,50 & 310,80 & 305,40 & NS & NS & NS & 98,30 \\
\hline $\begin{array}{l}\text { MMnd } \\
\left(n^{\circ} \text { día }^{-1}\right)\end{array}$ & 14304 & 18100 & 15229 & 15747 & 13643 & NS & NS & NS & 4767,1 \\
\hline $\begin{array}{l}\text { MMnb } \\
\left(n^{\circ} \text { bolo }^{-1}\right)\end{array}$ & 54,70 & 58,70 & 49,40 & 51,50 & 46,10 & $* *$ & NS & NS & 8,12 \\
\hline $\begin{array}{l}\text { TM } \\
\left(\text { s bolo-1) }^{-1}\right.\end{array}$ & 62,40 & 61,70 & 56,70 & 54,50 & 52,30 & $* *$ & NS & $*$ & 9,13 \\
\hline
\end{tabular}

${ }^{1}$ Lineal. ${ }^{2}$ Cuadrático. ${ }^{3}$ Tratamiento control $\times$ tratamientos con glicerina. ${ }^{4}$ Raiz cuadrada del error medio. ${ }^{*}<0,05 ; * *<0,01$, NS $=$ No significativo.

Fuente: Elaboración de los autores.

Tabla 6. Desempeño de bovinos alimentados con dietas con inclusión de hasta $30 \%$ de glicerina bruta bruta.

\begin{tabular}{|c|c|c|c|c|c|c|c|c|c|}
\hline \multirow{2}{*}{ Ítem } & \multicolumn{5}{|c|}{ Glicerina bruta (\%) } & \multicolumn{3}{|c|}{ Contrastes } & \multirow{2}{*}{$\mathrm{RMSE}^{4}$} \\
\hline & G0 & $\mathrm{G} 7,5$ & G15 & $\mathrm{G} 22,5$ & G30 & $\mathrm{L}^{1}$ & $\mathrm{C}^{2}$ & $0 \times \mathrm{GLI}^{3}$ & \\
\hline PC inicial, $\mathrm{kg}$ & 279,5 & 280,5 & 270,5 & 279,3 & 278,5 & ns & ns & ns & 4.46 \\
\hline PC final, kg & 413,9 & 427,6 & 423,1 & 427,3 & 403,5 & ns & ns & ns & 15.84 \\
\hline GMD, $\mathrm{kg} \mathrm{d}^{-1}$ & 1,54 & 1,69 & 1,75 & 1,70 & 1,44 & ns & ns & ns & 0.15 \\
\hline $\mathrm{G}: \mathrm{A}, \mathrm{kg} \mathrm{kg}^{-1}$ & 0,17 & 0,22 & 0,21 & 0,20 & 0,19 & ns & ns & ns & 0.02 \\
\hline
\end{tabular}

${ }^{1}$ Lineal. ${ }^{2}$ Cuadrático. ${ }^{3}$ Tratamiento control $\times$ tratamientos con glicerina. ${ }^{4}$ Raiz cuadrada del error medio. $*<0,05 ; * *<0,01, \mathrm{NS}=\mathrm{No}$ significativo. GMD = Ganancia diaria de peso, $\mathrm{G}: \mathrm{A}=$ Eficiencia alimenticia.

Fuente: Adaptado de Van Cleef et al. (2014).

\section{Conclusión}

La inclusión de hasta $30 \%$ de glicerina cruda en raciones de bovinos de la raza Nelore incrementa la eficiencia de alimentación, expresada en $\mathrm{g}$ FDN $h^{-1}$, aumenta la eficiencia de rumia en relación al FDN, disminuyendo el tiempo y el número de masticaciones merícicas por bolo ruminal, facilitando la ingestión de los alimentos e influenciando directamente en el tiempo de alimentación.

\section{Agradecimientos}

Los autores agradecen a la "Fundação de Apoio a Pesquisa do Estado de São Paulo" (FAPESP) por la financiación del proyecto de investigación y beca de estudio, y a Caramaru Alimentos S.A. por la oferta de parte de los ingredientes de las dietas utilizadas en esta investigación.

Todos los procedimientos utilizados en este estudio fueron aprobados por el "Comitê de Ética e Bem Estar Animal" de la Universidade Estadual Paulista - Unesp (Protocolo: 010707) y adoptando los principios del "Colegio Brasileiro de Experimentação Animal" (COBEA). 


\section{Referencias}

ABDAlla, A. L.; SILVA FILHO, J. C.; GODOI, A. R.; CARMO, C. A.; EDUARDO, J. L. P. Utilização de subprodutos da indústria de biodiesel na alimentação de ruminantes. Revista Brasileira de Zootecnia, Viçosa, MG, v. 37, p. 260-268, 2008. Especial.

BARROSO, D. S.; ALMEIDA, V. V. S.; SILVA, R. R.; OLIVEIRA, A. C.; ABREU FILHO, G.; LISBOA, M. M. Níveis crescentes de glicerina bruta na dieta de novilhas suplementadas a pasto: comportamento ingestivo. In: REUNIÃO ANUAL DA SOCIEDADE BRASILEIRA DE ZOOTECNIA, 47., 2010, Salvador. Anais... Salvador: SBZ, 2010. p. 1-3.

BÜRGER, P. J.; PEREIRA, J. C.; QUEIROZ, A. C; COELHO DA SILVA, J. F.; VALADARES FILHO, S. C.; CECON, P. R.; CASALI, A. D. P. Comportamento ingestivo em bezerros holandeses alimentados com dietas contendo diferentes níveis de concentrado. Revista Brasileira de Zootecnia, Viçosa, MG, v. 29, n. 1, p. 236242, 2000.

CARDOSO, A. R.; CARVALHO, S.; GALVANI, D. B.; PIRES, C. C.; GASPERIN, B. G.; GARCIA, R. P. A. Comportamento ingestivo de cordeiros alimentados com dietas contendo diferentes níveis de fibra em detergente neutro. Ciência Rural, Santa Maria, v. 36, n. 2, p. 604609, 2006

CHUNG, Y. H.; RICO, D. E.; MARTINEZ, C. M.; CASADY, T. W.; NOIROT, N.; AMES, A.; VARGA, G.A. Effects of feeding dry glycerin to early postpartum Holstein dairy cows on lactational performance and metabolic profiles. Journal of Dairy Science, Champaign, v. 90, n. 8 , p. 5682-5691, 2007.

DILORENZO, N.; GALYEAN, M. L. Applying technology with newer feed ingredients in feedlot diets. Do the old paradigms apply? Journal of Animal Science, Champaign, v. 88, n. 13, p. E123-E132, 2010.

DONKIN, S. S. Glicerol from biodiesel production: the new corn for dairy cattle. Revista Brasileira de Zootecnia, Viçosa, MG, v. 37, p. 280-286, 2008. Especial.

DONKIN, S. S.; KOSER, S. L.; WHITE, H. M.; DOANE, P. H.; CECAVA, M. J. Feeding value of glycerol as a replacement for corn grain in rations fed to lactating dairy cow. Journal of Dairy Science, Champaign, v. 92, n. 10, p. 5111-5119, 2009.

ELAM, N. A.; ENG, K. S.; BECHTEL, B.; HARRIS, J. M.; CROCKER, R. Glycerol from biodiesel production:considerations for feedlot diets. In: SOUTHWEST NUTRITION CONFERENCE, 23., 2008. Tempe. Proceedings... Tempe: University of Arizona, 2008. p. 131.
GOERING, H. G.; VAN SOEST, P. J. Forage fiber analysis (apparatus, reagents, procedures and some applications). Washington: Agricultural Research Service, 1970. 20 p.

LOPES, M. A.; MAGAlHÃES, G. P. Análise da rentabilidade da terminação de bovinos de corte em condição de confinamento: um estudo de caso. Arquivo Brasileiro de Medicina Veterinária e Zootecnia, Belo Horizonte, v. 57, n. 3, p. 374-379, 2005.

MACH, N.; BACH, A.; DEVANT, M. Effects of crude glycerin supplementation on performance and meat quality of Holstein bulls fed high-concentrate diets. Journal of Animal Science, Champaign, v. 87, n. 2, p. 632-638, 2009.

MENDONÇA, S. S.; CAMPOS, J. M. S.; VALADARES FILHO, S. C.; VALADARES, R. F. D.; SOARES, C. A.; LANA, R. P.; QUEIROZ, A. C.; ASSIS, A. J. E PEREIRA, M. L. A. Comportamento ingestivo de vacas leiteiras alimentadas com dietas à base de cana-de-açúcar ou silagem de milho. Revista Brasileira de Zootecnia, Viçosa, MG, v. 33, n. 3, p. 723-728, 2004.

MISSIO, R. L.; BRONDANI, I. L.; ALVES FILHO, D. C.; SILVEIRA, M. F.; FREITAS, L. S.; RESTLE, J. Comportamento ingestivo de tourinhos terminados em confinamento, alimentados com diferentes níveis de concentrado na dieta. Revista Brasileira de Zootecnia, Viçosa, MG, v. 39, n. 7, p. 1571-1578, 2010.

NATIONAL RESEARCH COUNCIL - NRC. Nutrient requirements of beef cattle. $7^{\text {th }}$ ed. Washington, D. C.: National Academy Press, 1996. 242 p.

PARSONS, G. L.; SHELOR, M. K.; DROUILLARD, J. S. Performance and carcass traits of finishing heifers fed crude glycerin. Journal of Animal Science, Champaign, v. 87, n. 2, p. 653-657, 2009.

PAZDIORA, R.; BRONDANI, I. L.; SILVEIRA, M. F.; ARBOITTE, M. Z.; CATTELAM, J.; PAULA, P. C. Efeitos da frequência de fornecimento do volumoso e concentrado no comportamento ingestivo de vacas e novilhas em confinamento. Revista Brasileira de Zootecnia, Viçosa, MG, v. 40, n. 10, p. 2244-2251, 2011.

SANTANA JUNIOR, H. A.; FIGUEIREDO, M. P.; SANTANA, E. O. C.; MENDES, F. B. L.; ABREU FILHO, G.; PINHEIRO, A. A.; LISBOA, M. M.; LUZ, Y. S.; VIANA, P. T.; FERREIRA, A. H. C.; RECH, C. L. S. Glicerina bruta na dieta de vacas lactantes mantidas em pastagem tropical: comportamento ingestivo. Semina: Ciências Agrárias, Londrina, v. 34, n. 3, p. 1339-1352, 2013.

SILVA, D. J.; QUEIROZ, A. C. Análise de alimentos: métodos químicos e biológicos. 3. ed. Viçosa, MG: 
Universidade Federal de Viçosa, MG, 2002. 235 p.

SILVA, R. R.; SILVA, F. F.; CARVALHO, G. G. P.; FRANCO, I. L.; VELOSO, C. M.; CHAVES, M. A.; BONOMO, P.; PRADO, I. N.; ALMEIDA, V. S. Comportamento ingestivo de novilhas mestiças de holandês x zebu confinadas. Archivos de Zootecnia, Córdoba, v. 54, n. 205, p. 75-85, 2005.

SILVA, R. R.; SILVA, F. F.; PRADO, I. N. Metodologia para o estudo do comportamento de bezerros confinados na fase de pós-aleitamento. Archivos Latinoamericanos de Producción Animal, Maracay, v. 14, n. 4, p. 135-138, 2006.
TREJO, C. O.; FAULKNER, D. B.; SHRECK, A.; HOMM, J. W.; NASH, T. G.; RODRIGUEZ-ZAS, S. L.; BERGER, L. L. Effects of co-products and breed of sire on the performance, carcass characteristics, and rates of ultrasound backfat and marbling deposition in feedlot cattle. Professional Animal Scientist, Charlottesville, v. 26, n. 6, p. 620-630, 2010.

VAN CLEEF, E. H. C. B.; EZEQUIEL, J. M. B.; D'AUREA, A. P.; FÁVARO, V. R.; SANCANARI, J. B. D. Crude glycerin in diets for feedlot Nelore cattle. Revista Brasileira de Zootecnia, Viçosa, MG, v. 43, n. 2, p. 86-91, 2014. 
